\title{
LACOURSIÈRE, Jacques et Claude BOUCHARD, Notre Histoire : Québec-Canada [an 1000-2000] - 15 volumes. Éditions Format, Montréal, 1972.1438 p., ill. \\ HOWARD, Richard, Jacques LACOURSIÈRE et Claude BOUCHARD, A New History of Canada - 15 volumes. Éditions Format, Montréal, 1973. 1438 p., ill.
}

\section{Micheline Johnson}

Volume 27, numéro 4, mars 1974

URI : https://id.erudit.org/iderudit/303314ar

DOI : https://doi.org/10.7202/303314ar

Aller au sommaire du numéro

Éditeur(s)

Institut d'histoire de l'Amérique française

ISSN

0035-2357 (imprimé)

1492-1383 (numérique)

Découvrir la revue

Citer ce compte rendu

Johnson, M. (1974). Compte rendu de [LACOURSIÈRE, Jacques et Claude BOUCHARD, Notre Histoire : Québec-Canada [an 1000-2000] - 15 volumes. Éditions Format, Montréal, 1972. 1438 p., ill. / HOWARD, Richard, Jacques LACOURSIÈRE et Claude BOUCHARD, A New History of Canada - 15 volumes. Éditions Format, Montréal, 1973. 1438 p., ill.] Revue d'histoire de l'Amérique française, 27(4), 590-593. https://doi.org/10.7202/303314ar d'utilisation que vous pouvez consulter en ligne. 
LACOURSIÈRE, Jacques et Claude Bouchard, Notre Histoire: Québec-Canada [an 1000-2000] - 15 volumes. Editions Format, Montréal, 1972. 1438 p. ill.

Howard, Richard, Jacques LACOURSIÈRE et Claude BouCHARD, A New History of Canada - 15 volumes. Editions Format, Montréal, 1973. 1438 p. ill.

Une histoire populaire du Canada, en 15 volumes, est actuellement disponible dans certaines chaînes de magasins d'alimentation. Cette série est publiée dans les deux langues et est due à la collaboration de quelques membres qui ont travaillé avec l'équipe du Boréal-Express. Le texte est de Jacques Lacoursière, l'iconographie est de Claude Bouchard, et Richard Howard a assuré la traduction anglaise de l'ouvrage après avoir assisté Lacoursière dans les sections concernant plus spécifiquement le Canada anglophone. Est-ce là une nouvelle tentative de présenter un "manuel unique" d'Histoire du Canada? Avant de répondre à cette question, nous examinerons quelque peu cette publication.

Chaque volume comprend une table des matières, 3 ou 4 chapitres, une liste de lectures suggérées et un tableau chronologique. Dans chacun, ces éléments sont répartis sur 96 pages, dont 85 de texte. L'ensemble propose donc une lecture de plus de 1,200 pages, parsemées de près de 1,100 illustrations variées. Le volume I est consacré à une description du territoire, à un exposé sur les tribus amérindiennes et au récit de l'exploration du Canada avant le XVII ${ }^{e}$ siècle. Le volume II, pour sa part, porte sur le XVII ${ }^{\mathrm{e}}$ siècle, alors que les volumes III et IV se concentrent sur le $\mathrm{XVIII}^{\mathrm{e}}$ siècle. Quatre volumes couvrent le $\mathrm{XIX}^{\mathrm{e}}$ siècle, et six le $\mathrm{XX}^{\mathrm{e}}$ siècle. Le volume $\mathrm{XV}$ en est un de prospective où sont cités un certain nombre de textes touchant les questions qui sont au centre des préoccupations actuelles des Canadiens. Ce dernier volume se termine par un index de 50 pages. L'accent, on le voit, est nettement mis sur l'histoire récente.

L'iconographie se caractérise avant tout par sa munificence. La couleur est utilisée avec art, chaque volume comprenant au moins 16 pages 
en couleur et chacune des autres pages étant imprimée en deux couleurs, ces couleurs elles-mêmes variant d'un volume à l'autre et, même, d'un cahier à l'autre. Quant au choix des illustrations, il est éclectique et d'inégale valeur. On trouve un très grand nombre de gravures, de dessins, de photos, de reproductions de peinture, d'objets d'art, de graphiques et de cartes. Les illustrations se caractérisent surtout par leur beauté ou leur caractère documentaire. Les thèmes québécois sont nettement majoritaires dans un ouvrage qui se veut surtout canadien: pour huit gravures de toutes sortes sur le Québec, on n'en trouve que cinq pour tout le reste du Canada dans les volumes X à XV. D'autre part, on reconnait, dans les premiers volumes, les illustrations des publications du Boréal-Express.

Cependant, une part importante de ces illustrations appelle certaines réserves. Il s'agit des cartes nombreuses qui figurent dans ces 15 volumes. En effet, plusieurs de ces cartes sont extrêmement difficiles à lire, étant donné qu'elles ne comportent ni échelle, ni légende. En fait, la plupart de ces cartes donnent l'illusion de la clarté. Quand on les analyse, on y découvre un grand nombre de lacunes de sorte que certaines sont moins des instruments de travail qu'un élément décoratif.

Jacques Lacoursière se tire habilement de la tâche ardue que pouvait représenter une rédaction simple mais complète, objective mais intéressante, de l'histoire canadienne. L'auteur y manifeste son aptitude à résumer d'une manière claire et pertinente les thèses les plus savantes de l'historiographie canadienne, comme celle de l'hypothèse de la venue de Vikings en Amérique. Il évite avec habileté de porter des jugements de valeur sur les personnages, comme nous y avaient habitués un certain nombre d'auteurs de manuels d'histoire. C'est ainsi que Cartier, Talon, Murray, Papineau, Macdonald et autres personnages figurent dans cette histoire sans recevoir automatiquement leur brevet de "héros national". Par ailleurs, l'ensemble des 15 volumes suit une trajectoire nettement politique car c'est la trame des événements politiques de l'histoire canadienne qui a servi d'épine dorsale à chacun de ces volumes. On a donc relégué à la fin de chaque volume, dans un chapitre de "civilisation", les événements d'ordre culturel, scientifique et social, qui se sont déroulés à chaque époque. De plus, comme l'annonce justement la préface, l'auteur cède "la parole aux témoins directs et aux acteurs des événements". En effet, les références aux documents sont nombreuses; elles le sont surtout, - trop, peut-être - lorsqu'il est question d'un événement brûlant de l'histoire canadienne. Enfin, le texte propose beaucoup d'informations sur toutes les parties du Canada. Est-ce dire qu'on a là une interprétation pan-canadienne de notre histoire? Il est difficile de répondre à cette question, tant l'auteur a évité de prendre parti dans un sens ou dans l'autre.

Cette apparente objectivité est cependant compromise par le choix des titres. En effet, certains titres et sous-titres nous paraissent parfois tendancieux. Ainsi la période qui va de 1603 à 1660 s'intitule "Une lutte qui commence"; la période qui va de 1672 à 1701: "Un affrontement inévitable"; le début de la campagne de 1754: "L'ouverture de la chasse"; l'affaire des Métis: "A l'ombre du gibet"; les réactions à la crise économi- 
que de 1929: "On défie le fédéral"; le centenaire de la Confédération: "Un long centenaire".

De toute manière, il est certainement peu commode, dans une collection de ce genre, d'avoir utilisé exclusivement des titres littéraires et non pas des titres semblables à ceux qu'on retrouve habituellement dans les ouvrages d'histoire. En effet, aucun personnage ou événement connus, n'est mentionné dans ces titres et ces sous-titres, de sorte qu'il est très difficile de retrouver un thème historique précis à travers le déroulement de l'ouvrage. Certes, il faut une certaine gymnastique intellectuelle pour faire le lien entre Une forte tentation et la Révolution américaine; les Problèmes de communication et la construction des chemins de fer intercontinentaux; L'ère des banquets et la période qui a précédé la Confédération; Une question de bateaux et la période de l'immigration au début du XXe siècle; L'Ouest et à l'Est et le gouvernement de Diefenbaker. A notre sens, l'auteur a abusé de ces sous-titres à connotation très contemporaine qui rendront la consultation de ces volumes encore plus difficile dans quelques années, alors que les lecteurs ne pourront absolument plus rapprocher ces sous-titres des événements qui les ont inspirés en 1972.

Dans les chapitres sur la civilisation, les sous-titres peuvent davantage prêter à confusion. En effet, ces titres sont choisis pour des raisons mystérieuses car le contenu de chacun de ces chapitres n'a que des liens très indirects avec le titre qui le précède. Parfois même le titre ne correspond pas du tout à la période; ainsi le titre "Vive le libéralisme", relatif à la période 1841-1864, ne comporte pas une seule allusion aux luttes idéologiques du XIX ${ }^{\mathrm{e}}$ siècle; de même, "Tout en images" a été choisi pour décrire le développement de la civilisation de 1939 à 1950, c'est-à-dire justement l'ère qui a précédé l'avènement de la télévision. Ce n'est donc pas le texte même de cet ouvrage d'histoire du Canada qui peut être critiqué mais bien davantage certains aspects de sa présentation. Car ces volumes sont finalement très difficiles à consulter si on n'a pas déjà une idée du déroulement de l'histoire. Qui plus est, même l'index ne nous est d'aucun secours, puisqu'il ne renvoie pas à la tomaison et que l'ensemble des volumes est paginé de 1 à 1438. Fait à souligner également: l'index français est 2 fois plus dense que l'index anglais.

Pourtant, le texte anglais semble suivre de près le texte original. Mais il est clair que la version anglaise n'est pas une traduction au sens technique du mot. Car on note à de nombreux endroits des modifications au texte de Lacoursière. Cela s'explique par le fait que c'est un historien versé dans les ouvrages de vulgarisation qui a assumé la traduction et non pas un traducteur professionnel. Ces modifications de détails semblent être dues davantage aux problèmes de la mise en pages qu'à une présentation différente des faits. En fait, à première lecture, les événements-crises de l'histoire canadienne, sur lesquels les historiens se sont rarement entendus, tels la déportation des Acadiens, la conquête, les troubles de 1837-38, l'Union, la Confédération, l'affaire Riel, les écoles séparées du Manitoba, la Conscription de 1917, etc. sont décrits le plus froidement possible, et principalement au moyen de citations documentaires, ce qui laisse pressentir une 
grande objectivité. Mais la version anglaise présente souvent des différences. Ainsi, à la fin du paragraphe sur Cabot, on ajoute la phrase suivante: "Wherever he did come ashore, on one island or the other, he planted the arms of Henri VII Tudor (...)." A partir de 1760, les textes cités sont presque toujours différents. Cela est particulièrement frappant pour les épisodes de Murray (IV: 304-308), de Craig (V: 403-406), de la Confédération (VII: 593-597); dans le cas de Riel (VII: 650-654) les différences de citations peuvent changer complètement l'interprétation de l'événement. En dernière analyse, il faut constater que chaque version présente à son lecteur l'interprétation de son groupe national plutôt qu'une juxtaposition d'interprétations divergentes. Cela est particulièrement évident lorsqu'on examine les sous-titres anglais, qui sont moins orientés, sans être plus utiles cependant.

Il y a près de 25 ans, L'Action nationale publiait un numéro spécial "Pour ou contre le manuel d'histoire unique ?" à la suite du débat lancé aux Communes par T.-D. Bouchard. La situation a bien changé depuis. Les anciens manuels d'histoire ont été mis au rancart; la science historique a fait d'énormes progrès; deux enquêtes ont examiné la question de l'enseignement de l'histoire nationale, et de nouvelles méthodes sont apparues mettant l'accent sur la méthodologie historique, plus que sur l'enseignement nationaliste. Mais les paroles de Groulx, dans ce numéro spécial, restent toujours d'actualité: "Si l'on admet l'existence de deux nationalités au Canada, et que chacune, par conséquent, a bel et bien sa conscience historique, chacune doit posséder son histoire à soi. Ce qui n'implique nullement que les deux histoires doivent s'affronter avec hostilité." (L'Action nationale, mai 1950 , p. 348). L'histoire du Canada dont nous venons de parler, illustre de nouveau, à sa manière un peu subtile l'existence des "deux solitudes". 